\title{
Resolución de problemas y motivación en espacios virtuales. Propuesta de una línea de investigación.
}

\author{
Sara Ballestero Madorrán \\ parasara790hotmail.com \\ Universidad de Salamanca
}

\section{Introducción}

El presente artículo, propone una posible línea de investigación en el terreno de la Resolución de Problemas que de una forma muy incipiente se ha desarrollado en el Trabajo de Grado "Resolución de Problemas y Motivación en Espacios Virtuales" y que sin duda necesita de las aportaciones y esfuerzos de nuevos y más rigurosos procesos de investigación que la desarrollen.

A pesar de que desde mi posición como autora de este Trabajo de Grado no es fácil admitirlo, ni quizá lo más adecuado, su valor se sitúa en su dimensión procesual más que productiva, en el sentido de que -y muy a mi pesar- no se han obtenido novedosos hallazgos capaces de revolucionar la práctica educativa de RP en el contexto de los Espacios Virtuales. Sin embargo, el proceso de investigación es en sí mismo enriquecedor ya que las deficiencias y carencias que, sin duda, se hacen patentes en "Motivación y Resolución de Problemas en Espacios Virtuales" han supuesto el cuestionamiento de los procesos de investigación no como un ejercicio de censura sino de mejora, lo que es imprescindible para retomar un futuro estudio en éste ámbito.

\section{El punto de partida}

La enseñanza de la Matemática ha desempeñado y desempeña un importante papel dentro de los currículos, y por tanto, del corpus educativo a nivel internacional. Sin embargo, la importancia que atribuyen los distintos países a dicha materia es variable. Una de las aspiraciones o desafíos educativos de E. E. U. U., por poner un ejemplo, es situarse el número uno en la escala de rendimiento, considerándolo predictor de un rendimiento académico general de más alto nivel.

Por tanto, aunque globalmente se está de acuerdo en la importancia de la educación matemática, no hay unicidad de pensamiento y opiniones en cuanto a qué, cómo y cuándo debe enseñarse. Pero a pesar de ello, tal y como apuntan los expertos, la importancia de la matemática estriba en su utilidad para la vida práctica, su fomento de habilidades cognoscitivas y su lenguaje, elocuente per se.

El conocimiento matemático incluye una serie de conceptos; conceptos complejos ya que a ellos se asocian contenidos, procesos y actitudes, que tienen aun mayor importancia para el desarrollo autónomo de la persona, lo que constituye uno de los objetivos fundamentales de los Sistemas Educativos.

Los contenidos, se refieren a conceptos o nociones como asimetría, discontinuidad, etc. que tienen significados específicos en el campo de las matemáticas, y que se extrapolan a la vida cotidiana en la que la comprensión de su significado es muy funcional; procesos como la demostración, implícita en las Matemáticas, adquieren un valor semántico y operativo que las transciende; las actitudes, hacen alusión a los hábitos o maneras del alumno al enfrentarse a las matemáticas, como la objetividad, el rigor...y que se requieren para hacer buenas matemáticas. 
Dentro de la generalidad de la enseñanza de la Matemática debemos encuadrar el campo específico de la Resolución de Problemas (RP). Por tanto, es importante tener en cuenta el rol de todos estos aspectos descritos e incluidos en la RP, uno de los pilares de la línea de investigación que proponemos.

En este sentido, es importante que se encuentren nuevas vías y se redefinan las ya existentes en este campo de estudio, para la mejora de la adquisición de estrategias de RP, donde -entre otras- entra en juego una importante variable que también se convierte en objeto de estudio de la línea propuesta, nos referimos a la motivación.

Este complejo constructo cumple el doble y poderoso papel de aliado y/o enemigo en materia de enseñanza, ya que interfiere directamente en los procesos de enseñanza-aprendizaje, y por tanto en los niveles de desarrollo alcanzados por el alumno.

No todo termina en este punto, ya que hasta ahora hemos delimitado dos de los pilares fundamentales de la línea de investigación, sin embargo nos queda el tercero de ellos, no por ello menos importante, ya que por otro lado constituye el contexto donde situamos los procesos involucrados tanto en la RP como en la motivación. Nos referimos al terreno de los Espacios Virtuales.

Como resultado del increíble desarrollo del hardware/software así como de las redes de información y, en definitiva del desarrollo de los sistemas multimedia, se pone a nuestra disposición una nueva realidad potencialmente educativa; ello, sin embargo, no significa que se cierren las puertas de la enseñanza tradicional ya que ésta no deja de cumplir un papel fundamental y más cuando se trata de enseñanza en niveles obligatorios. Sin embargo, estando como estamos inmersos en la Sociedad de la Información, no debemos volver la espalda a estos nuevos contextos y seguir dando motivos para que se mantenga la ya "archiconocida" sentencia de "la escuela ha perdido el tren".

Además, y ya para concluir con este asunto, es importante anotar que, a priori, la legitimidad de un estudio centrado en la RP desde la perspectiva educativa, y por tanto también la legitimidad de esta línea de estudio, estriba en la posibilidad de su aplicabilidad práctica una vez éste haya concluido.

\section{Cuestiones Metodológicas}

En referencia a las cuestiones metodológicas, son muchos los expertos en el tema que apuntan desde hace un tiempo la aparición de cambios significativos en la investigación de los procesos de RP -y de investigación educativa en general- respecto a sus inicios, que coinciden con la primera mitad del siglo XX, y que se caracteriza por el énfasis en la experimentación. Este énfasis en la experimentación viene de la mano del desarrollo del método científico, del que la Física obtuvo excelentes resultados y que pronto encontró aplicabilidad en otros campos del saber. No obstante en el caso de las Ciencias Humanas, la coincidencia de sujeto y objeto de conocimiento limita las posibilidades de este eficaz método en lo que respecta a la aplicación de determinados métodos de observación.

La aludida coincidencia de su objeto/ sujeto de estudio, además, supone la aceptación del carácter transitorio y contextualizado del conocimiento en esta área. Esta parcela del saber, por otro lado, esta abierta a las influencias de otras áreas como la Psicología, la Sociología, la Matemática...lo que no supone la pérdida de su propia especificidad, basada en su preocupación en el estudio de los modos de enseñanza y aprendizaje de los procesos de RP.

Estas observaciones han provocado en los últimos años, la proliferación de literatura centrada en la evolución de la investigación educativa en general, tratando de legitimarla para estimular y aumentar la unidad y la productividad en estos dominios. Este tipo de bibliografía pone un énfasis especial en el asunto del método y la metodología.

Tal y como apunta Vallecillos (Carrillo Yáñez, J., 1998) método es "cualquier conjunto de acciones ordenadas con las que pretendemos conseguir unos resultados" y metodología "la aplicación coherente de un método"; sin embargo, la elección del método y la aplicación de la metodología está mediatizada por las tradiciones y los paradigmas de la educación. 
En nuestra propuesta, optamos por la elección del paradigma (de influencia francófona) basado en una teoría interna, que tiene como línea base la tentativa de conformación de un cuerpo teórico coherente que nos permita desenvolvernos con esquemas adecuados en la especificad de nuestro campo (el de la RP). Esta teoría interna surgirá, como apunta José Manuel Matos (Carrillo Yáñez, J. 1998), del resultado de un proceso de perfeccionamiento conceptual que se procura adecuar al estudio de los procesos empíricos; en este caso es la confrontación con teorías generalistas sobre la educación (Procesos cognitivos, de RP, motivación y educación en espacios virtuales ), cuya finalidad es el establecimiento de una determinada didáctica de la RP, coherente con los procesos y constructos analizados y con la especificad del conocimiento matemático.

Por otro lado, este paradigma que consideramos adecuado para guiar nuestra propuesta de investigación, se encuadra dentro de la tradición pedagógica que procura divulgar métodos de enseñanza eficaces ya que su finalidad es fomentar comportamientos adecuados en el alumno. Para ello es (y nos es) de gran utilidad el saber acumulado como resultado de la reflexión sobre la práctica, específico de dicha tradición. Sin embargo el paradigma elegido no está exento de cierto carácter ecléctico, ya que también toma elementos de las tradiciones de la ciencia empírica y la filosófica escolástica, que junto a la pedagógica constituyen las tres grandes tradiciones de la investigación en la RP. La adopción del paradigma de la teoría interna nos permitirá por tanto la adopción de métodos de distinta naturaleza dada su flexibilidad. Teniendo en cuenta la apertura y libertad de movimientos que supone la elección de éste paradigma, a continuación hacemos referencia a nuestro esquema metodológico, basado en las ideas de Vallecillos (Carrillo Yáñez, J., 1998), que afirma que "un correcto enfoque metodológico de la investigación educativa comporta el análisis detallado de los siguientes elementos mínimos: objetivos que se persiguen, instrumento de recogida de datos y análisis".

\section{Los objetivos y la definición del problema}

En referencia a los objetivos que han guiado la elaboración de "Resolución de problemas y Motivación en espacios virtuales" y que esperamos que sean tomados en cuenta en futuras investigaciones centradas en este campo, es preciso hacer mención a distintos aspectos que a pesar de su especificidad, se relacionan muy estrechamente entre sí.

La Definición del problema supone el primero de los pasos así como el establecimiento del problema de investigación, ya que muchas veces éste no está claro y puede obstaculizar el transcurso de la misma. A grandes rasgos, la meta la investigación es el análisis conjunto de los Procesos de RP, Motivación, y Espacios Virtuales, para establecer un conjunto de pautas que aúnen sus potencialidades y contribuyan a mejorar dichos procesos, mediante el estudio del diseño hipermedia orientado a la RP.

\section{El estado de la cuestión}

En segundo lugar es imprescindible adentrarse en un estudio teórico previo, para determinar el Estado de la cuestión, que en este caso constituirá una parte fundamental de la investigación, y en el que se hace imprescindible emplear bastante tiempo y esfuerzo, ya que se debe realizar un análisis del tema desde distintas perspectivas, científica, filosófica, histórica y didáctica... dependiendo del constructo específico y del momento en que se esté analizando centrándose más en una u otra perspectiva. Todo ello con una pretensión clara, esto es, situar el problema de investigación en un marco teórico y científico adecuado.

En este sentido aparece el primer problema, ya que no existen antecedentes de investigación específicos de nuestro objeto de estudio (RP aritméticos verbales y Motivación en Espacios virtuales) y es preciso redefinir los marcos teóricos ya existentes y crear nuestro propio marco de trabajo, puesto que todos ellos adolecen de la adecuación requerida. En este sentido, debemos hacer un intento de conceptuación propio. 
En el caso de la Resolución de Problemas, aspecto central de la línea de investigación propuesta, en el que existen distintas perspectivas explicativas de los procesos seguidos por los individuos, optamos por una postura híbrida, ya que incluyéndola dentro del marco de la Psicología Cognitiva, la configuramos principalmente tomando elementos de la Psicología del Procesamiento de la Información, las distintas teorías de los esquemas, así como de la Teoría del Aprendizaje Mediado que vinculamos especialmente a los Espacios Virtuales-. Por otro lado, también destaca la importancia atribuida a la motivación, sin olvidar conectarlo con la virtualidad en un multimarco teórico que interrelacione los tres aspectos conjuntamente.

Figura 1. Multimarco Teórico propuesto

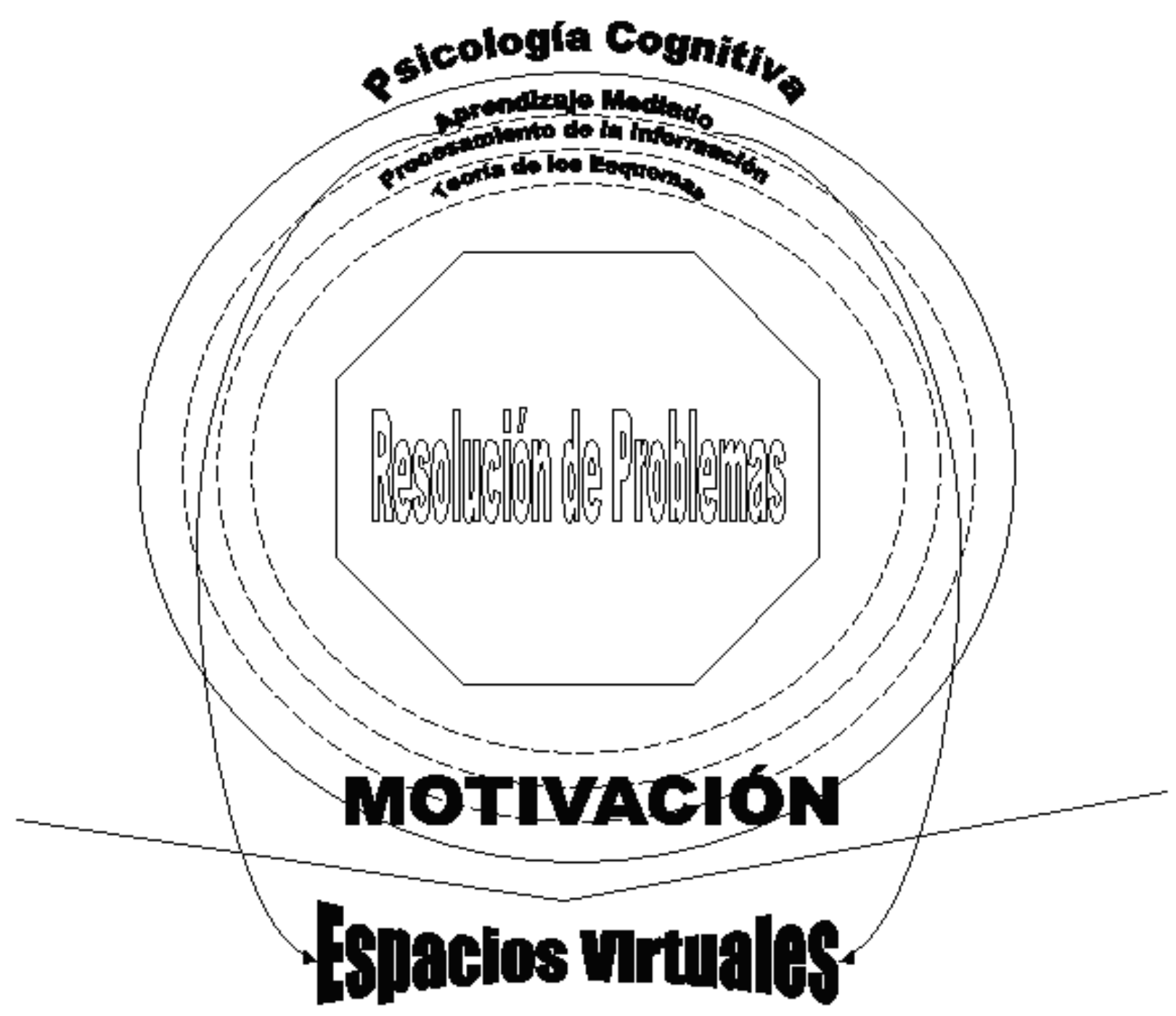

\section{La determinación de la hipótesis}

En cuanto a la Determinación de hipótesis, en primer lugar, debemos clarificar que lo que nosotros formulamos es una hipótesis de trabajo, es decir, en principio, se plantea como guía de la investigación, y no para ser sometida a una verificación experimental. Formulamos nuestra hipótesis general de la siguiente manera:

Si aunamos las potencialidades educativas de la motivación y de los espacios virtuales y las aplicamos al área de la RP y a sus propias especificidades, se potenciarán y mejorarán los procesos de aprendizaje de los individuos en esta área.

No obstante, la línea de investigación propuesta se concibe como el primer paso en el que se 
contempla la fundamentación teórica y el diseño previo de un proyecto hipermedia orientado a la RP aritméticos verbales para, posteriormente, en una futuras investigaciones, llevar a cabo el desarrollo y la producción de dicho proyecto hipermedia con el propósito de realizar un estudio profundo _en contextos escolares específicos_ sobre los procesos de aprendizaje en RP mediados por recursos hipermedia.

Una vez formulada la hipótesis, establecemos como variables fundamentales de la propuesta de investigación, tal y como anunciamos con anterioridad:

1. Los procesos de RP (y específicamente los problemas aritméticos verbales)

2. La motivación

3. La virtualidad

\section{La obtención de datos}

En cuanto a la obtención de datos, es importante tener en cuenta tanto las fuentes bibliográficas como el material Web o fuentes procedentes de la Red. A partir de las mismas, y teniendo presentes los objetivos de la línea de investigación, se llevará a cabo el análisis, guiado por el paradigma de la Teoría interna, anteriormente aludido, cuya pretensión última es la mejora de la práctica educativa.

\section{Bibliografía}

CASTRO, E. (Editor) (2001) Didáctica de la matemática en la Educación Primaria. Madrid, Síntesis.

LESTER JR., F. Y GAROFALO, J. (1982) Mathematical Problem Solving. Issues in Research. Philadelphia, Pennsylvania, The Franklin Institute Press.

MARSHALL P.S. (1995) Schemas in Problem Solving. New York, Cambridge University Press.

MORENO, M. F., Socas, M. y Rodino J. D. (Editores) (2002) Investigación en Educación Matemática. Quinto Simposio de la Sociedad Española de Investigación en Educación Matemática. Almería, Universidad de Almería.

NICKERSON, R. PERKINS, D. \& SMITH, E. (1994) Enseñar a Pensar. Aspectos de la aptitud intelectual. Barcelona, Paidós.

NCTM (1992) Handbook of Research on Mathematics Teaching and Learnig. New York.

NCTM (1996) Comunicación in Mathematics. K-12 and Beyond. Editor University of Massachusetts at Amherst.

POLYA, G. (1962) Mathematical discovery: On understanding, learning, and teaching problem solving. (Vol. 1). Nueva Cork, Wiley \& Sons.

RESNICK, L. B.Y FORD, W. W. (1990) La enseñanaza de las matemáticas y sus fundamentos psicológicos. Barcelona, Ediciones Paidós.

RIVIERE, A. (1987). El sujeto de la psicología cognitiva. Alianza.

SCHUNK, D. H. (1997). Teorías del aprendizaje. México, Pertince-Hall Hispanoamericana, S.A.

THE COGNITION \& TECHNOLOGY GROUP AT VANDERBILT (1997) The Jasper Proyect. London, Lawrence Erlbaum Associates, Publishers. 
